\title{
Controlling the bias error of Fokker- Planck methods for rarefied gas dynamics simulations
}

Cite as: Phys. Fluids 31, 062005 (2019); https://doi.org/10.1063/1.5097884

Submitted: 29 March 2019 . Accepted: 21 May 2019 . Published Online: 14 June 2019

Patrick Jenny, Stephan Küchlin, and Hossein Gorji
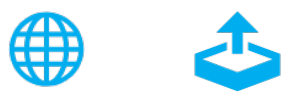

View Online

Export Citation

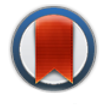

CrossMark

\section{ARTICLES YOU MAY BE INTERESTED IN}

A dusty gas model-direct simulation Monte Carlo algorithm to simulate flow in microporous media

Physics of Fluids 31, 062007 (2019); https://doi.org/10.1063/1.5094637

Particle number control for direct simulation Monte-Carlo methodology using kernel estimates

Physics of Fluids 31, 062008 (2019); https://doi.org/10.1063/1.5097902

On the concept and theory of induced drag for viscous and incompressible steady flow Physics of Fluids 31, 065106 (2019); https://doi.org/10.1063/1.5090165

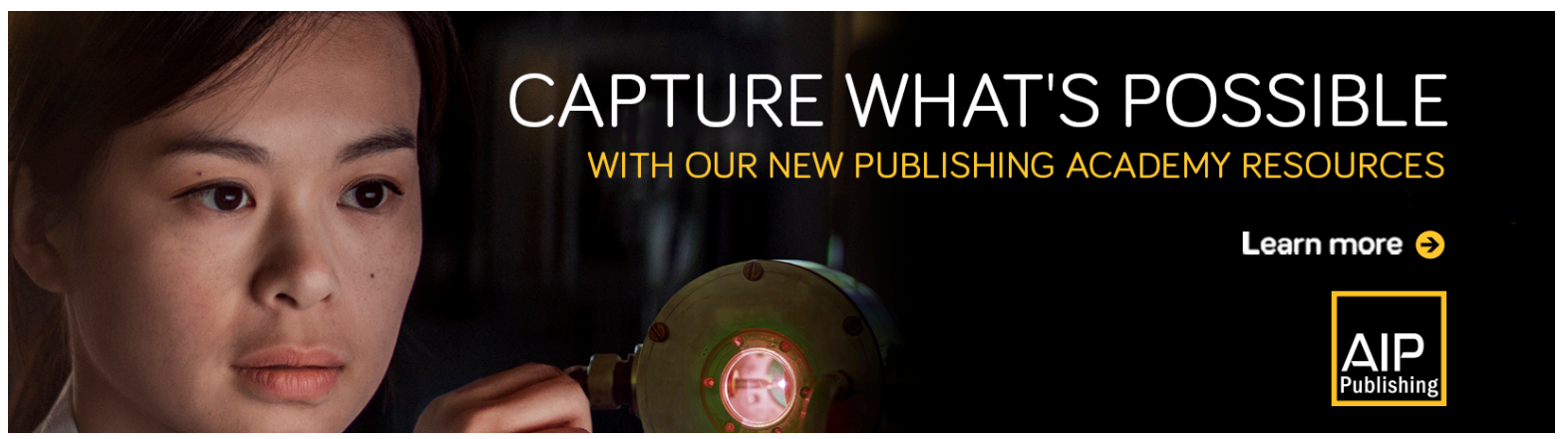




\title{
Controlling the bias error of Fokker-Planck methods for rarefied gas dynamics simulations
}

\author{
Cite as: Phys. Fluids 31, 062005 (2019); doi: 10.1063/1.5097884 \\ Submitted: 29 March 2019 - Accepted: 21 May 2019 • \\ Published Online: 14 June 2019
}

Patrick Jenny, ${ }^{1, a)}$ Stephan Küchlin, ${ }^{1, b)}$ and Hossein Gorji ${ }^{2, c)}$

\author{
AFFILIATIONS \\ ${ }^{1}$ Institute of Fluid Dynamics, ETH Zurich, Sonneggstrasse 3, CH-8092 Zurich, Switzerland \\ ${ }^{2}$ Computational Mathematics and Simulation Science, EPFL, MA C2 642, CH-1015 Lausanne, Switzerland
}

Note: This paper is part of the special issue on Direct Simulation Monte Carlo-The Legacy of Graeme A. Bird.

a) Electronic mail: jenny@ifd.mavt.ethz.ch

b) Electronic mail: kuechlin@ifd.mavt.ethz.ch

${ }^{c}$ Electronic mail: mohammadhossein.gorji@epfl.ch

\begin{abstract}
Direct simulation Monte-Carlo (DSMC) is the most established method for rarefied gas flow simulations. It is valid from continuum to near vacuum, but in cases involving small Knudsen numbers (Kn), it suffers from high computational cost. The Fokker-Planck (FP) method, on the other hand, is almost as accurate as DSMC for small to moderate Kn, but it does not have the computational drawback of DSMC, if Kn is small [P. Jenny, M. Torrilhon, and S. Heinz, "A solution algorithm for the fluid dynamic equations based on a stochastic model for molecular motion," J. Comput. Phys. 229, 1077-1098 (2010) and H. Gorji, M. Torrilhon, and P. Jenny, "Fokker-Planck model for computational studies of monatomic rarefied gas flows," J. Fluid Mech. 680, 574-601 (2011)]. Especially attractive is the combination of the two approaches leading to the FP-DSMC method. Opposed to other hybrid methods, e.g., coupled DSMC/Navier-Stokes solvers, it is relatively straightforward to couple DSMC with the FP method since both are based on particle solution algorithms sharing the same data structure and having similar components. Regarding the numerical accuracy of such particle methods, one has to distinguish between spatial truncation errors, time stepping errors, statistical errors and bias errors. In this paper, the bias error of the FP method is analyzed in detail, and it is shown how it can be reduced without increasing the particle number to an exorbitant level. The effectiveness of the discussed bias error reduction scheme is demonstrated for uniform shear flow, for which an analytical reference solution was derived.
\end{abstract}

Published under license by AIP Publishing. https://doi.org/10.1063/1.5097884

\section{INTRODUCTION}

While the Navier-Stokes Fourier system accurately describes compressible gas flows, if the mean free path length between molecular collisions remains extremely small compared to any geometrical length scale of interest, these equations fail to be predictive in cases where this is not the case. For such high Knudsen number (Kn) gas flows, which occur under rarefied conditions or in very small geometries, the Boltzmann equation has to be considered. It describes the evolution of the molecular velocity probability density function (PDF) and has to be solved in a high dimensional space (e.g., in 7D; three dimensions for the velocity sample space, three dimensions for the physical space, and one dimension for time). Due to the curse of dimensionality and due to the integral molecular collision term, typical continuum methods like finite-volume, finite-element, and finite-difference methods are in general not suited for this task, and therefore, often particle methods are used instead. Nevertheless high fidelity schemes can be constructed by the direct approach, where the probability density is discretized in the whole phase space (see, e.g., Ref. 3). Accordingly, spectral methods have been developed for computing the collision operator. ${ }^{4-6}$

An $a b$ inito molecular based description of rarefied gas flows is provided by molecular dynamics (MD), which considers the interactions between all molecules in a fluid. ${ }^{7}$ However, stiff spatiotemporal resolutions and the enormous amount of required computational particles make its application only suitable for extremely small problems.

Direct simulation Monte Carlo (DSMC) pioneered by Bird, on the other hand, requires a much smaller number of computational particles than there are molecules. It is based on two 
fractional steps, i.e., a streaming step, in which the particles are evolved in physical space, and a collision step, in which collisions are calculated for randomly selected particle pairs. DSMC proved to be extremely powerful and is widely used to solve scientific and technical rarefied gas dynamics (RGD) problems. ${ }^{10-13}$ It is accurate for all $\mathrm{Kn}$ and much more efficient than MD. However, since the size of the computational grid cells has to be chosen in the order of the mean free path length, and since the number of computational particles per cell has to be reasonably large, DSMC becomes extremely expensive for small $\mathrm{Kn}$ (though still much cheaper than MD). ${ }^{14,1}$ Moreover, the time step size has to be small enough to resolve the mean free time. Note that typically, these spatial and temporal resolution requirements are much more severe than those imposed by variations of macroscopic quantities.

Motivated by the high computational cost of DSMC for low Kn gas flow simulations, a Fokker-Planck (FP) collision operator was introduced as an approximation of the integral collision term in the Boltzmann equation. It consists of a drift term and a diffusion term in the velocity sample space, and substituting it into the Boltzmann equation one obtains a FP equation. Already, Kirkwood ${ }^{16}$ and Kirkwood et al. ${ }^{17}$ introduced a Fokker-Planck model for liquids, and Lebowitz et al. ${ }^{18}$ showed that the velocity distribution function for the Fokker-Planck equation matches well with the Boltzmann distribution for small Knudsen numbers, i.e., close to equilibrium. It was shown by Pawula and Gradiner that for any continuous stochastic Markov process, there exists a FP equation, which describes the evolution of the corresponding PDF. In the case considered here, the velocity PDF is governed by a jump process, but if the mean free time is small enough (i.e., if $\mathrm{Kn}$ is small enough), the discontinuous velocity process can be well approximated by a continuous one. Therefore, it is not surprising that the FP model is very accurate for small to moderate $\mathrm{Kn}$ and less predictive for large Kn. Main advantage of the FP method is its computational efficiency. Unlike in DSMC, it is not necessary to resolve mean free path length and mean free time scale in FP simulations since an accurate time integration scheme could be devised requiring to resolve spatial and temporal variations of only macroscopic quantities. First, a FP collision operator with linear drift was proposed. ${ }^{1}$ For various test cases with $\mathrm{Kn}$ up to about two, it was demonstrated that obtained velocity distribution, molecular stresses, and drag were comparable with experiments and DSMC results. As analytically shown, however, the linear drift model results in wrong heat fluxes and thus cannot accurately predict temperature distributions. This Prandtl number problem could be fixed with the introduction of a cubic drift model, ${ }^{2,19}$ which was demonstrated for various challenging test cases. ${ }^{20}$ This issue is also discussed in the recent work by Singh et al., ${ }^{21}$ in which they proposed a way to fix the Prandtl number resulting from the Fokker-Planck equation. They achieved that by introducing an extra streaming in the particle position to fix the transport properties including the Prandtl number. However, their modification requires evaluation of the temperature gradient and hence does not reproduce correct relaxation rates of heat fluxes in a space-independent scenario. Later, the FP model was extended for mixtures ${ }^{22}$ and polyatomic gas, ${ }^{23-26}$ and a noise reduction scheme for low Mach numbers was devised. ${ }^{27}$ In this context also, an improved wall kernel was developed, ${ }^{19}$ which accounts for internal energy modes. ${ }^{22,28}$

To take advantage of both the accuracy of DSMC for large Kn and the efficiency of FP for small Kn, a framework to combine the two methods was proposed. ${ }^{29,30}$ Since both FP and DSMC solution algorithms are based on evolving computational particles, it is possible to switch between the two methods on a cell-by-cell and a time-step-by-time-step basis. This FP-DSMC method was tested for various difficult test cases, e.g., for a Laval nozzle flow expanding into vacuum, which is challenging, since $\mathrm{Kn}$ varies from very small inside the nozzle (continuum condition) to very large (near vacuum condition). It was shown that the obtained results were essentially identical to those from DSMC, but could be obtained at a small fraction of the cost. ${ }^{30}$ Recently, a general purpose FP-DSMC code for complex geometries, adaptive grid refinement, and load balancing was developed. ${ }^{3}$

Particle methods used for FP simulations and DSMC suffer from four types of numerical errors, i.e., (i) from time stepping errors, (ii) spatial discretization errors, (iii) statistical errors, and

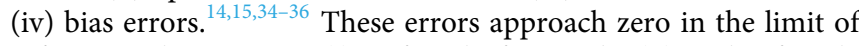
infinitesimal time steps (i), infinitely fine grids, (ii) and infinitely many particles (iii) and (iv), respectively. Recently, a particle time integration scheme for the FP method was devised ${ }^{1}$ to deal with errors associated with (i). It allows to take time steps much larger than the mean free time scale. In fact, for the FP model with linear drift, it is statistically exact for stationary homogeneous cases. Practically, this means that only spatial and temporal variations of macroscopic quantities have to be resolved, but not mean free path and mean free time. In order to reduce errors associated with (ii), it was proposed to interpolate macroscopic quantities appearing in the stochastic particle evolution equations from grid cells to the estimated particle locations after half a time step. Errors associated with (iii) can simply be reduced by averaging the results from multiple independent (with different random seeds) simulations of the same case, or, if statistically stationary, by time averaging. Interestingly, however, such averaged results do not converge to those obtained from single simulations with increased numbers of particles. This difference is related to (iv), i.e., it is termed bias error.

As mentioned previously, most particle methods suffer from bias errors in one way or another. For example, in PDF, methods used to model turbulent reactive flows bias errors were investigated, and in order to reduce them, a hybrid finite-volume/particle method was devised as a solution algorithm. ${ }^{34,35,37}$ This approach indeed proved to be extremely effective, i.e., in some cases, the computational cost to achieve a specified level of accuracy could be reduced by almost two orders of magnitude. As those transported PDF methods for turbulent reactive flows also aim to solve a Fokker-Planck equation, a similar hybrid approach could be envisioned for the FP method for RGD. In this paper, however, exponentially weighted time averaging (EWTA) is investigated for bias error reduction.

The paper is structured as follows. In Sec. II, the FP method for RGD is briefly revisited and its necessary basics are explained. Section III deals with an analysis of the bias error and its origin, and in Sec. IV, EWTA for bias error reduction is explained. Numerical studies of bias errors in uniform shear flow are presented in Sec. V and the effect of EWTA is demonstrated. The paper concludes with Sec. VI.

\section{THE FOKKER-PLANCK METHOD FOR RAREFIED GAS DYNAMICS}

As mentioned in Sec. I, DSMC is a very powerful method to compute nonequilibrium gas flows, and as shown, e.g., in 
Ref. 38, converged DSMC results are consistent with solutions of the Boltzmann equation. If the Boltzmann equation is written as

$$
\frac{\partial \mathscr{F}}{\partial t}+v_{i} \frac{\partial \mathscr{F}}{\partial x_{i}}+\frac{\partial a_{i} \mathscr{F}}{\partial v_{i}}=S_{B}
$$

where $\boldsymbol{v}=\left(v_{1}, v_{2}, v_{3}\right)^{T}$ and $\boldsymbol{x}=\left(x_{1}, x_{2}, x_{3}\right)^{T}$ are velocity sample space coordinates and physical space coordinates, $\mathscr{F}=\mathscr{F}(\boldsymbol{v}, \boldsymbol{x}, t)$ the mass density function, i.e., the gas density in the $\boldsymbol{v}-\boldsymbol{x}$-space, $\boldsymbol{a}=\left(a_{1}, a_{2}, a_{3}\right)^{T}$ an external acceleration, and $S_{B}$ the Boltzmann collision source term accounting for redistribution of mass in the $\boldsymbol{v}$-space. The macroscopic gas flow quantities, i.e., density, velocity, molecular stresses, and temperature are obtained as

$$
\begin{aligned}
\rho(\boldsymbol{x}, t) & =\int_{\mathbb{R}^{3}} \mathscr{F}(\boldsymbol{v}, \boldsymbol{x}, t) d \boldsymbol{v}, \\
\boldsymbol{U}(\boldsymbol{x}, t) & =\frac{1}{\rho(\boldsymbol{x}, t)} \int_{\mathbb{R}^{3}} \mathscr{F}(\boldsymbol{v}, \boldsymbol{x}, t) \boldsymbol{v} d \boldsymbol{v}, \\
p_{i j}(\boldsymbol{x}, t) & =P_{i j}(\boldsymbol{x}, t)-\rho(\boldsymbol{x}, t) U_{i}(\boldsymbol{x}, t) U_{j}(\boldsymbol{x}, t),
\end{aligned}
$$

and

respectively, where

$$
\theta(\boldsymbol{x}, t)=\frac{p_{i i}(\boldsymbol{x}, t)}{\rho(\boldsymbol{x}, t)} \frac{m}{3 k}
$$

$$
P_{i j}(\boldsymbol{x}, t)=\int_{\mathbb{R}^{3}} \mathscr{F}(\boldsymbol{v}, \boldsymbol{x}, t) v_{i} v_{j} d \boldsymbol{v} .
$$

Here, $d \boldsymbol{v}=d v_{1} d v_{2} d v_{3}$ and in the whole paper, wherever the same index appears twice in a term, Einstein's summation convention is applied. Furthermore, $m$ is the molecular mass and $k$ the Boltzmann constant. DSMC proved to be accurate for all Knudsen numbers, i.e., in the continuum limit as well as for near vacuum. For small Knudsen numbers, however, it becomes very expensive since spatial and temporal resolution requirements are directly linked to the mean free path length and the mean free time. For example, there exist many flow problems where $\mathrm{Kn}$ is very small, but nonequilibrium effects still play an important role, or where continuum and rarefied regions coexist. For such cases, the Fokker-Planck method was developed.

The Fokker-Planck method is based on the FP equation

$$
\frac{\partial \mathscr{F}}{\partial t}+v_{i} \frac{\partial \mathscr{F}}{\partial x_{i}}+\frac{\partial a_{i} \mathscr{F}}{\partial v_{i}}=S_{F P}
$$

with, e.g.,

$$
S_{F P}=-\frac{\partial}{\partial v_{i}}\left(\frac{1}{\tau}\left(U_{i}-v_{i}\right) \mathscr{F}\right)+\frac{\partial^{2}}{\partial v_{i} \partial v_{i}}\left(\frac{\theta k}{\tau m} \mathscr{F}\right) .
$$

The two terms of the FP collision operator (8) describe drift and diffusion of $\mathscr{F}$ in the $\boldsymbol{v}$-space. It is evident that Eq. (7) is obtained by replacing $S_{B}$ in the Boltzmann equation (1) with $S_{F P}$, and like the Boltzmann collision operator, $S_{F P}$ conserves mass, momentum, and energy. Furthermore, the time scale $\tau$ can be chosen such that the molecular stresses evolve correctly as described by the Boltzmann equation.

Like DSMC, due to the curse of dimensionality, the FP method relies on a particle solution algorithm. Concretely, the mass density function is represented by a cloud of particles as

$$
\mathscr{F}(\boldsymbol{v}, \boldsymbol{x}) \approx \overline{\mathscr{F}}^{N}(\boldsymbol{v}, \boldsymbol{x})=\sum_{k=1}^{N}\left\{M^{(k)} \delta\left(\boldsymbol{v}-\boldsymbol{V}^{(k)}\right) \delta\left(\boldsymbol{x}-\boldsymbol{X}^{(k)}\right\},\right.
$$

and if consistent, then $\mathscr{F}(\boldsymbol{v}, \boldsymbol{x})=\mathbb{E}\left[\overline{\mathscr{F}}^{N}(\boldsymbol{v}, \boldsymbol{x})\right]$, where the operator $\mathbb{E}[\cdot]$ denotes expectation. Each of the $N$ particles has an index $k$, a mass $M^{(k)}$ (total gas mass divided by $N$ ), a position $\boldsymbol{X}^{(k)}$ in physical space, and a molecular velocity $\boldsymbol{V}^{(k)}$. Note that for $N \rightarrow \infty$, the mass density $\mathscr{F}$ in the $\boldsymbol{v}-\boldsymbol{x}$-space is exactly represented by the particle density $\overline{\mathscr{F}}^{N}$. Furthermore, if in addition the particles evolve as described by the stochastic differential equations

$$
d X_{i}^{(k)}=V_{i}^{(k)} d t
$$

and

$$
d V_{i}^{(k)}=a_{i} d t+\frac{d t}{\tau}\left(U_{i}-V_{i}^{(k)}\right)+\sqrt{\frac{2 \theta k}{\tau m}} d W_{i}^{(k)},
$$

then $\tilde{\mathscr{F}}^{N}=\mathscr{F}$ obeys the FP equation (7) with (8). ${ }^{39-4}$

The crucial advantage of the FP method for RGD compared to DSMC is that the relaxation time scale $\tau$, which is proportional to the mean free time, does not have to be resolved, if the particle positions and velocities are evolved as

$$
\begin{aligned}
V_{i}^{n+1}-V_{i}^{n}= & -\left(1-e^{-\Delta t / \tau}\right)\left(V_{i}^{n}-U_{i}-a_{i} \tau\right) \\
& +\sqrt{\frac{C^{2}}{B}} \xi_{1, i}+\sqrt{A-\frac{C^{2}}{B}} \xi_{2, i}
\end{aligned}
$$

and

$$
\begin{aligned}
X_{i}^{n+1}-X_{i}^{n}= & \left(1-e^{-\Delta t / \tau}\right)\left(V_{i}^{n}-U_{i}-a_{i} \tau\right) \tau \\
& +\left(U_{i}+a_{i} \tau\right) \Delta t+\sqrt{B} \xi_{1, i}
\end{aligned}
$$

with

$$
\begin{gathered}
A=\frac{\theta k}{m}\left(1-e^{-2 \Delta t / \tau}\right), \\
B=\frac{\theta k \tau^{2}}{m}\left(\frac{2 \Delta t}{\tau}-\left(1-e^{-\Delta t / \tau}\right)\left(3-e^{-\Delta t / \tau}\right)\right),
\end{gathered}
$$

and

$$
C=\frac{\theta k \tau}{m}\left(1-e^{-\Delta t / \tau}\right)^{2}
$$

where $\xi_{1, i}$ and $\xi_{i, 2}$ are independent normally distributed random variables with zero mean and a variance of one. ${ }^{1}$ Note that the superscript $(k)$ was omitted to improve readability. Instead, the superscripts $n+1$ and $n$ were introduced denoting the new and old time levels, respectively; $\Delta t=t^{n+1}-t^{n}$ is the time step size. It can be proven that the particle integration scheme (12) and (13) is statistically exact for any time step size $\Delta t$, if $\tau, \boldsymbol{a}, \boldsymbol{U}$, and $\theta$ are independent of $\boldsymbol{x}$ and $t$. Practically, this means that only these macroscopic quantities have to be spatially and temporally resolved, i.e., opposed to DSMC, it is not necessary to resolve mean free path and mean free time. The advantage of this approach has been demonstrated for a large variety of relevant flows and up to $\mathrm{Kn} \approx 2$ the FP method proved to deliver accurate velocity fields. ${ }^{2,20}$ It has to be mentioned, however, that opposed to DSMC the scheme (12) and (13) honors momentum and energy conservation only statistically. Thus, a disadvantage of models based on Langevin equations is that additional noise gets introduced, which also affects the bias error.

Since with the linear drift term in the FP collision operator (8), it is not possible to also obtain the correct heat fluxes an improved 
FP collision operator with a cubic drift term was devised. ${ }^{2}$ Here, for simplicity, we focus on $S_{F P}$ given by Eq. (8). The FP method for RGD was also generalized for mixtures and polyatomic gas, and a variance reduction scheme was devised to cope with the stochastic noise, which is, in particular, problematic in low Mach number flow simulations. ${ }^{24,25,27}$ The most important development, however, is the combination of the FP method with DSMC. ${ }^{30,31,42}$ The resulting FPDSMC method offers unique advantages in simulating gas flows where continuum and rarefied regions coexist. Such cases are extremely challenging to simulate since Navier-Stokes solvers can only appropriately describe gas flow in thermodynamic equilibrium, i.e., in general only if the Knudsen number is very small and thus when DSMC is exorbitantly expensive. Several attempts have been made to couple Navier-Stokes solvers with DSMC, but this is problematic due to coupling issues between continuum and particle solvers, and since there exists a wide range of small $\mathrm{Kn}$ where nonequilibrium effects still can play a role. ${ }^{43}$ Therefore it is often unclear where to put the boundary between Navier-Stokes and DSMC domains. Coupling the FP method with DSMC is much less problematic since there exists quite a large range in which $\mathrm{Kn}$ is small enough that the FP method is accurate and large enough that DSMC is still efficient. Therefore, and since both methods share the same data structure, one can simply switch between the two collision operators; i.e., in each cell and every time step one can determine the cell Knudsen number (mean free path length divided by the cell width), and if it is smaller than one, then $S_{F P}$ and otherwise $S_{B}$ is applied. It was demonstrated, for example, that FP-DSMC of gas flow through a Laval nozzle expanding into vacuum is up to 40 times faster than pure DSMC, while the results are virtually indistinguishable. ${ }^{30}$ This particular test case is representative for satellite attitude controllers, but many other examples exist, where the FP-DSMC method provides a unique advantage.

Like DSMC, the particle method developed for the FP method suffers from time stepping errors, spatial discretization errors, statistical errors and bias errors. While time stepping errors and spatial discretization errors can be kept small by applying the scheme (12) and (13) and by employing a grid and time step size fine enough to resolve the macroscopic quantities, statistical errors and bias errors can be severe, unless a huge number of particles is employed. While statistical errors can be reduced by averaging independent simulation results (i.e., obtained with different random seeds), or, if statistically stationary, by averaging over a time window, the bias error is more problematic. The latter is discussed in Sec. III.

\section{ANALYSIS OF BIAS ERROR}

To analyze the bias error arising in FP simulations, only the velocity sample space is considered here. Without external acceleration nor transport in physical space the Langevin equation (11) reduces to the Ornstein-Ulenbeck (OU) process

$$
d V_{i}^{(k)}=\frac{d t}{\tau}\left(U_{i}-V_{i}^{(k)}\right)+\sqrt{\frac{2 \theta k}{\tau m}} d W_{i}^{(k)},
$$

and the mass density function $\mathscr{F}(\boldsymbol{v}, t)$ is governed by the FP equation

$$
\frac{\partial \mathscr{F}}{\partial t}=\underbrace{-\frac{\partial}{\partial v_{i}}\left(\frac{1}{\tau}\left(U_{i}-v_{i}\right) \mathscr{F}\right)+\frac{\partial^{2}}{\partial v_{i} \partial v_{i}}\left(\frac{\theta k}{\tau m} \mathscr{F}\right)}_{S_{F P}} .
$$

Based on the conservation properties of $S_{F P}$, it directly follows that $\rho(t)=\rho, \boldsymbol{U}(t)$, and $\theta(t)$ are constant in time (therefore, for simplicity and without loss of generality, a constant $\rho$ can be assumed in the following derivations). The molecular stresses, on the other hand, relax toward $\rho \theta \mathrm{km}^{-1} \delta_{i j}$, i.e.,

$$
\begin{aligned}
\frac{\partial p_{i j}}{\partial t}=\frac{\partial P_{i j}}{\partial t} & =\frac{2}{\tau}\left(\frac{\rho \theta k}{m} \delta_{i j}-p_{i j}\right) \\
& =\frac{2}{\tau}\left(\frac{P_{l l}}{3} \delta_{i j}-P_{i j}\right)-\frac{2 \rho}{\tau}\left(\frac{U_{l} U_{l}}{3} \delta_{i j}-U_{i} U_{j}\right) \\
& =\frac{2}{\tau}\left(\frac{p_{l l}}{3} \delta_{i j}-p_{i j}\right) .
\end{aligned}
$$

In order to study the bias errors related to $U$ and $\boldsymbol{p}$, we consider a cloud of $N$ particles with index $k$ and estimate the macroscopic quantities as

$$
\begin{aligned}
& \boldsymbol{U} \approx \overline{\boldsymbol{U}}^{N}=\frac{1}{N} \sum_{k=1}^{N} \boldsymbol{V}^{(k)}, \\
& P_{i j} \approx \bar{P}_{i j}^{N}=\frac{\rho}{N} \sum_{k=1}^{N} V_{i}^{(k)} V_{j}^{(k)},
\end{aligned}
$$

and

$$
p_{i j} \approx \bar{p}_{i j}^{N}=\bar{P}_{i j}^{N}-\rho \bar{U}_{i}^{N} \bar{U}_{j}^{N} .
$$

Note that $\overline{\boldsymbol{U}}^{N}, \bar{P}_{i j}^{N}$, and $\bar{p}_{i j}^{N}$ are random numbers with the expectations

$$
\begin{aligned}
& \mathbb{E}\left[\overline{\boldsymbol{U}}^{N}\right]=\boldsymbol{U}, \\
& \mathbb{E}\left[\bar{P}_{i j}^{N}\right]=P_{i j},
\end{aligned}
$$

and

$$
\mathbb{E}\left[\bar{p}_{i j}^{N}\right]=P_{i j}-\rho \mathbb{E}\left[\bar{U}_{i}^{N} \bar{U}_{j}^{N}\right],
$$

respectively, i.e., there is no bias in estimating $\boldsymbol{U}$ and $\boldsymbol{P}$, but in general there is one in estimating $\boldsymbol{p}$.

Next, we consider for each particle the stochastic difference equation

$$
V_{i}^{(k)^{n+1}}=V_{i}^{(k)^{n}}+\frac{\Delta t}{\tau}\left(\bar{U}_{i}^{N^{n}}-V_{i}^{(k)^{n}}\right)+\sqrt{\frac{2 \bar{p}_{l l}^{N^{n}} \Delta t}{3 \rho \tau}} \xi_{i}^{(k)^{n+1}} .
$$

Note that $\xi_{i}^{(k)^{n+1}}$ are independent normally distributed random variables with $\mathbb{E}\left[\xi_{i}^{(k)^{n+1}}\right]=0$ and $\mathbb{E}\left[\xi_{i}^{(k)^{n+1}} \xi_{j}^{(k)^{n+1}}\right]=\delta_{i j}$. Furthermore, we introduce

$$
\begin{gathered}
\bar{\xi}_{i}^{N}=\frac{1}{N} \sum_{k=1}^{N} \xi_{i}^{(k)}, \\
\bar{\Xi}_{i j}^{N}=\frac{1}{N} \sum_{k=1}^{N} \xi_{i}^{(k)} \xi_{j}^{(k)},
\end{gathered}
$$

and

$$
\bar{\xi}_{i j}^{N}=\frac{1}{N} \sum_{k=1}^{N} \xi_{j}^{(k)} V_{i}^{(k)} .
$$

The evolution of $\bar{U}_{i}^{N}$ according to Eq. (26) reads

$$
\bar{U}_{i}^{N^{n+1}}=\bar{U}_{i}^{N^{n}}+\underbrace{\frac{\Delta t}{\tau}\left(\bar{U}_{i}^{N^{n}}-\bar{U}_{i}^{N^{n}}\right)}_{0}+\sqrt{\frac{2 \bar{p}_{l l}^{N^{n}} \Delta t}{3 \rho \tau}} \bar{\xi}_{i}^{N^{n+1}},
$$


and the expectation of $\bar{U}_{i}^{N^{n+1}}$ is

$$
\begin{aligned}
\mathbb{E}\left[\bar{U}_{i}^{N^{n+1}}\right] & =\mathbb{E}\left[\bar{U}_{i}^{N^{n}}\right]+\mathbb{E}\left[\sqrt{\frac{2 \bar{p}_{l l}^{N^{n}} \Delta t}{3 \rho \tau}}\right] \underbrace{\mathbb{E}\left[\bar{\xi}_{i}^{N^{n+1}}\right]}_{0} \\
& =\mathbb{E}\left[\bar{U}_{i}^{N^{n}}\right],
\end{aligned}
$$

i.e., there is no bias error associated with the evolution of $\overline{\boldsymbol{U}}^{N}$. To investigate the bias error associated with the evolution of $\bar{P}_{i j}^{N}$, we first multiply Eq. (26) with $V_{j}^{(k)^{n+1}}$ and obtain

$$
\begin{aligned}
V_{i}^{(k)^{n+1}} V_{j}^{(k)^{n+1}}= & V_{i}^{(k)^{n}} V_{j}^{(k)^{n}}+\frac{\Delta t}{\tau}\left(\bar{U}_{i}^{N^{n}} V_{j}^{(k)^{n}}+\bar{U}_{j}^{N^{n}} V_{i}^{(k)^{n}}\right. \\
& \left.-2 V_{i}^{(k)^{n}} V_{j}^{(k)^{n}}\right)+\left(V_{i}^{(k)^{n}} \xi_{j}^{(k)^{n}}+V_{j}^{(k)^{n}} \xi_{i}^{(k)^{n}}\right) \sqrt{\frac{2 \bar{p}_{l l}^{N^{n}} \Delta t}{3 \rho \tau}} \\
& +\frac{\Delta t^{2}}{\bar{\tau}^{N^{2}}}\left(\bar{U}_{i}^{N^{n}}-V_{i}^{(k)^{n}}\right)\left(\bar{U}_{j}^{N^{n}}-V_{j}^{(k)^{n}}\right)+\frac{\Delta t}{\tau}\left(\bar{U}_{i}^{N^{n}}\right. \\
& -V_{i}^{\left.(k)^{n}\right) \xi_{j}^{(k)^{n}}} \sqrt{\frac{2 \bar{p}_{l l}^{N^{n}} \Delta t}{3 \rho \tau}}+\frac{\Delta t}{\tau}\left(\bar{U}_{j}^{N^{n}}-V_{j}^{(k)^{n}}\right) \xi_{i}^{(k)^{n}} \\
& \times \sqrt{\frac{2 \bar{p}_{l l}^{N^{n}} \Delta t}{3 \rho \tau}}+\frac{2 \bar{p}_{l l}^{N^{n}} \Delta t}{3 \rho \tau} \xi_{i}^{(k)^{n}} \xi_{j}^{(k)^{n}} .
\end{aligned}
$$

Then, we sum over all $N$ realizations, divide by $N$, multiply with $\rho$, and in the limit of $\Delta t \rightarrow 0$, we obtain

$$
\begin{aligned}
\frac{\bar{P}_{i j}^{N^{n+1}}-\bar{P}_{i j}^{N^{n}}}{\Delta t}= & \frac{2}{\tau}\left(\frac{\bar{P}_{l l}^{N^{n}}}{3} \bar{\Xi}_{i j}^{N^{n}}-\bar{P}_{i j}^{N^{n}}\right)-\frac{2 \rho}{\tau}\left(\frac{\bar{U}_{l}^{N^{n}} \bar{U}_{l}^{N^{n}}}{3} \bar{\Xi}_{i j}^{N^{n}}-\bar{U}_{i}^{N^{n}} \bar{U}_{j}^{N^{n}}\right) \\
& +\left(\bar{\xi}_{i j}^{N}+\overline{\xi U}_{j i}^{N}\right) \sqrt{\frac{2 \rho \bar{p}_{l l}^{N^{n}}}{3 \tau \Delta t}} .
\end{aligned}
$$

The expectation of Eq. (33) is

$$
\begin{aligned}
\frac{\partial P_{i j}}{\partial t} & =\frac{2}{\tau}\left(\frac{P_{l l}}{3} \delta_{i j}-P_{i j}\right)-\frac{2 \rho}{\tau} \mathbb{E}\left[\frac{\bar{U}_{l}^{N} \bar{U}_{l}^{N}}{3} \delta_{i j}-\bar{U}_{i}^{N} \bar{U}_{j}^{N}\right] \\
& =\frac{2}{\tau}\left(\frac{\mathbb{E}\left[\bar{p}_{l l}^{N}\right]}{3} \delta_{i j}-\mathbb{E}\left[\bar{p}_{i j}^{N}\right]\right)
\end{aligned}
$$

and the difference between Eqs. (19) and (34) leads to the equation

$$
\begin{aligned}
\frac{\partial \varepsilon_{i j}}{\partial t}= & \frac{2 \rho}{3 \tau} \mathbb{E}\left[\left(\bar{U}_{l}^{N}-U_{l}\right)\left(\bar{U}_{l}^{N}-U_{l}\right)\right] \delta_{i j} \\
& -\frac{2 \rho}{\tau} \mathbb{E}\left[\left(\bar{U}_{i}^{N}-U_{i}\right)\left(\bar{U}_{j}^{N}-U_{j}\right)\right]
\end{aligned}
$$

of the bias error $\varepsilon_{i j}$ associated with the numerical evolution of $P_{i j}$. From this simple analysis, it becomes evident that the bias error of the molecular stresses can be reduced, if better approximations of the mean velocity are used, e.g., if $N$ is increased.

\section{EWTA FOR BIAS ERROR REDUCTION}

From a practical viewpoint, unfortunately, the number of particles which can be employed is always limited due to computational cost and memory requirements. This is the motivation for consistent hybrid finite-volume/particle methods, where the noisy estimates
$\bar{U}_{i}^{N}$ are replaced by the consistent, but much less noisy finite volume fields $U_{i}^{F V} \cdot{ }^{34,35}$ In PDF simulations of turbulent reactive flows, it was shown that such hybrid finite-volume/particle methods indeed can reduce the bias error dramatically ${ }^{37}$ and nothing speaks against the idea of applying the same approach for RGD. Here, however, bias error reduction based on EWTA is investigated. Obviously, time averaging of the macroscopic quantities used in the particle evolution equations is much less memory intensive than increasing $N$, and in general also computationally more efficient. Moreover, time averaging also reduces the statistical error. Note, however, that EWTA, which is explained in more detail next, is only applicable to statistically stationary cases.

As discussed in Sec. III, the estimate $\bar{U}_{i}^{N}$ of the gas velocity component $U_{i}$ is a random variable with a standard deviation (statistical error) proportional to $N^{-1 / 2}$. But if $V_{i}^{(k)}(t)$ obeys a stationary stochastic process, instead of simply increasing $N$ also time averaging can be employed to reduce statistical errors and bias-errors, i.e.,

$$
\begin{aligned}
U_{i}^{n} \approx\left(\bar{U}_{i}^{N, M}\right)^{n} & =\frac{1}{M} \sum_{m=n-M+1}^{n}\left(\bar{U}_{i}^{N}\right)^{m} \\
& =\frac{1}{M N} \sum_{m=n+1-M}^{n} \sum_{k=1}^{N}\left(V_{i}^{(k)}\right)^{m} .
\end{aligned}
$$

Note that $\lim _{M \rightarrow \infty}\left(\bar{U}_{i}^{N, M}\right)^{n}=U_{i}$, for all $N>0$, and that in the ideal case where $\forall m: \mathbb{E}\left[V_{(i)}^{m} V_{(i)}^{m-1}\right] \equiv U_{(i)} U_{(i)}$, the statistical error of $\left(\bar{U}_{i}^{N, M}\right)^{n}$ is the same as that of $\left(\bar{U}_{i}^{N M}\right)^{n}$ (indices in brackets indicate that Einstein's summation convention is suppressed). Furthermore, note that

$$
\left(\bar{U}_{i}^{N, M+1}\right)^{n+1}=\underbrace{\frac{1}{M+1}}_{1-\mu}\left(\bar{U}_{i}^{N}\right)^{n+1}+\underbrace{\frac{M}{M+1}}_{\mu}\left(\bar{U}_{i}^{N, M}\right)^{n},
$$

which is much simpler to implement. The memory factor $\mu \in[0,1]$ can also be fixed, which leads to the EWTA scheme

$$
\left(\bar{U}_{i}^{N, \mu}\right)^{n+1}=(1-\mu)\left(\bar{U}_{i}^{N}\right)^{n+1}+\mu\left(\bar{U}_{i}^{N, \mu}\right)^{n}
$$

with the integral time averaging constant $T=-\Delta t / \ln (\mu)(\Delta t$ is the time step size). In inhomogeneous simulations, EWTA can be applied in each cell $\Omega$, but since the number of particles in $\Omega$ varies, it is advised to also apply EWTA for $N$, i.e.,

$$
\left(\bar{N}^{\mu}\right)^{n+1}=(1-\mu) N^{n+1}+\mu\left(\bar{N}^{\mu}\right)^{n},
$$

and to compute $\bar{U}_{i}^{N, \mu}$ as

$$
\left(\bar{U}_{i}^{N, \mu}\right)^{n+1}=\frac{(1-\mu)\left(N \bar{U}_{i}^{N}\right)^{n+1}+\mu\left(\bar{N}^{\mu} \bar{U}_{i}^{N, \mu}\right)^{n}}{\left(\bar{N}^{\mu}\right)^{n+1}} .
$$

EWTA has been proposed previously, but here for the first time, it is systematically investigated in the context of the FP method for RGD.

\section{NUMERICAL STUDIES}

To investigate the bias error resulting from the FP method, evolution of molecular stresses in uniform shear flow is considered. This simple test case is highly relevant, as relaxation of second moments 
in nonequilibrium shear flows is largely responsible for the accuracy of RGD models and solution algorithms; also in predicting more complicated flow scenarios. Furthermore, the test case is simple enough that we could derive an analytical solution, and therefore, there is no uncertainty regarding reference with which the numerical results are compared. Also important is the fact that this flow can be simulated in zero spatial dimensions (note that reduction of uniform shear flow to a homogeneous relaxation problem has often been used in the literature for a variety of reasons ${ }^{44,45}$ ) and that a statistically exact particle velocity integration scheme could be devised. This rules out spatial discretization and time stepping errors. Furthermore, the statistical error was reduced to a very low level as all the presented results are averages over a huge number of independent simulations (with different random seeds). All this allowed us to systematically study the bias error in almost complete isolation. Numerical studies illustrate convergence as the number of particles gets increased and show the effectiveness of EWTA to obtain accurate steady state solutions despite small particle numbers.

\section{A. Analytical reference-Uniform shear flow}

Uniform shear flow with

$$
\begin{aligned}
\nabla \boldsymbol{U}=\boldsymbol{A} & =\left[\begin{array}{lll}
\partial U_{1} / \partial x_{1} & \partial U_{1} / \partial x_{2} & \partial U_{1} / \partial x_{3} \\
\partial U_{2} / \partial x_{1} & \partial U_{2} / \partial x_{2} & \partial U_{2} / \partial x_{3} \\
\partial U_{3} / \partial x_{1} & \partial U_{3} / \partial x_{2} & \partial U_{3} / \partial x_{3}
\end{array}\right] \\
& =\left[\begin{array}{lll}
0 & S & 0 \\
0 & 0 & 0 \\
0 & 0 & 0
\end{array}\right]
\end{aligned}
$$

is considered. Note that by definition in uniform shear flow, all second and higher moments are constant in space, but not the mean velocity components, rather their first spatial derivatives. Therefore the matrix $\boldsymbol{A}$ has nonzero components, i.e., the gas velocity varies in space.

It will be shown that an analytical solution for the first two velocity moments resulting from the Langevin equation

$$
d V_{i}^{(k)}=-\underbrace{\left(\frac{1}{\tau}+\frac{1}{\tau^{\prime}}\right)}_{\tau_{e}^{-1}}\left(V_{i}^{(k)}-U_{i}\right) d t+\sqrt{\frac{2 \theta k}{\tau m}} d W_{i}^{(k)}
$$

can be derived. The superscript $(k)$, which refers to the particle with index $k$, will be omitted in the following derivations. Note that the term $-\left(V_{i}-U_{i}\right) d t / \tau^{\prime}$ in Eq. (42) is an energy sink to allow for steady state solutions (thus $\tau_{e}$ is the effective drift time scale) and that Eqs. (17) and (42) are identical for $\tau^{\prime} \rightarrow \infty$. Since the gas velocity gradient and all second and higher velocity moments are invariant in space, it is convenient to work with a Lagrangian equation for the fluctuating velocity $\boldsymbol{V}^{\prime}=\boldsymbol{V}-\boldsymbol{U}$. Therefore, we first derive an equation which describes the evolution of the gas velocity $\boldsymbol{U}$ along a molecular path. While the gas velocity equation

$$
\frac{\partial U_{i}}{\partial t}+U_{l} \frac{\partial U_{i}}{\partial x_{l}}=-\frac{1}{\rho} \frac{\partial p_{i l}}{\partial x_{l}}
$$

is equivalent to

$$
\underbrace{\frac{\partial U_{i}}{\partial t}+V_{l} \frac{\partial U_{i}}{\partial x_{l}}}_{\frac{d U_{i}(X(t), t)}{d t}}=\left(V_{l}-U_{l}\right) \frac{\partial U_{i}}{\partial x_{l}}-\frac{1}{\rho} \frac{\partial p_{i l}}{\partial x_{l}},
$$

the latter has a stochastic component, i.e., the left-hand side describes how $\boldsymbol{U}$ changes as one moves through physical space with the molecular velocity $\boldsymbol{V}$. Note that due to homogeneity, the last term becomes zero and this equation reduces to

$$
d U_{i}=A_{i l}\left(V_{l}-U_{l}\right) d t .
$$

Taking the difference between Eqs. (42) and (45), one arrives at the evolution equation for the fluctuating velocity components $V_{i}^{\prime}=V_{i}$ - $U_{i}$ of a nominal molecule, which reads

$$
d V_{i}^{\prime}=-A_{i l} V_{l}^{\prime} d t-\underbrace{\left(\frac{1}{\tau}+\frac{1}{\tau^{\prime}}\right)}_{\tau_{e}^{-1}} V_{i}^{\prime} d t+\sqrt{\frac{2 \theta k}{\tau m}} d W_{i}
$$

To derive the evolution of the molecular stresses, the consistent difference equation

$$
V_{i}^{\prime n+1}=V_{i}^{\prime n}-A_{i l} V_{l}^{\prime n} \Delta t-\frac{\Delta t}{\tau_{e}} V_{i}^{\prime n}+\sqrt{\frac{2 \theta k \Delta t}{\tau m}} \xi_{i}^{n+1}
$$

is multiplied with $\rho V_{j}^{\prime n+1} / \Delta t$ and the expectation of the result (for $\Delta t$ $\rightarrow 0$ ) reads

$$
\frac{d p_{i j}}{d t}=-\underbrace{\left(A_{i l} p_{l j}+A_{j l} p_{l i}\right)}_{S B_{i j}}-\alpha p_{i j}+\alpha \Lambda \delta_{i j}
$$

with

$$
\begin{aligned}
\alpha & =\frac{2}{\tau_{e}}, \\
\Lambda & =\frac{\rho \theta k}{m} \frac{\tau_{e}}{\tau},
\end{aligned}
$$

and

$$
\boldsymbol{B}=\left[\begin{array}{ccc}
2 p_{12} & p_{22} & p_{23} \\
p_{22} & 0 & 0 \\
p_{23} & 0 & 0
\end{array}\right]
$$

Equivalently, one can write

$$
\begin{gathered}
\frac{\partial p_{23}}{\partial t}=-\alpha p_{23}, \\
\frac{\partial p_{22}}{\partial t}=-\alpha p_{22}+\alpha \Lambda \\
\frac{\partial p_{33}}{\partial t}=-\alpha p_{33}+\alpha \Lambda, \\
\frac{\partial p_{13}}{\partial t}=-\alpha p_{13}-S p_{23} \\
\frac{\partial p_{12}}{\partial t}=-\alpha p_{12}-S p_{22}
\end{gathered}
$$

and

$$
\frac{\partial p_{11}}{\partial t}=-\alpha p_{11}-2 S p_{12}+\alpha \Lambda
$$

for the individual components. Note that in general solutions of this system involve varying temperatures $\theta$. To derive an analytical 
reference solution of $\boldsymbol{p}(t)$, however, we require that $\Lambda=\rho \theta k \tau_{e} /(m \tau)$ remains constant, which implies that $\theta=p_{i i} m /(3 \rho k)=\theta_{\text {ref }}$; later, we determine under which conditions this is the case. One can verify that under this assumption the solution is given by the analytical expressions

$$
\begin{aligned}
& p_{23}(t)=p_{23}(0) e^{-\alpha t}, \\
& p_{22}(t)=\left(p_{22}(0)-\Lambda\right) e^{-\alpha t}+\Lambda, \\
& p_{33}(t)=\left(p_{33}(0)-\Lambda\right) e^{-\alpha t}+\Lambda, \\
& p_{13}(t)=\left(p_{13}(0)-S t p_{23}(0)\right) e^{-\alpha t}, \\
& p_{12}(t)=-\frac{S}{\alpha} \Lambda+\left(S \Lambda\left(t+\frac{1}{\alpha}\right)+p_{12}(0)-S p_{22}(0) t\right) e^{-\alpha t},
\end{aligned}
$$

and

$$
\begin{aligned}
p_{11}(t)= & \left(S^{2} p_{22}(0)-S^{2} \Lambda\right) t^{2} e^{-\alpha t}-\left(2 S p_{12}(0)+2 \frac{S^{2}}{\alpha} \Lambda\right) t e^{-\alpha t} \\
& +\left(p_{11}(0)-\Lambda\left(1+2 \frac{S^{2}}{\alpha^{2}}\right)\right) e^{-\alpha t}+\Lambda\left(1+2 \frac{S^{2}}{\alpha^{2}}\right)
\end{aligned}
$$

As mentioned above, we are interested in solutions with $p_{i i}(t)$ $=3 \Lambda \tau / \tau_{e}(\forall t \geq 0)$, which is only possible, if $p_{i i}(0)=3 \Lambda \tau / \tau_{e}$. On the other hand, the condition that $\lim _{t \rightarrow \infty} p_{i i}(t)=3 \Lambda+\Lambda S^{2} \tau_{e}^{2} / 2=p_{i i}(0)$ leads to the relationship

$$
\tau=\left(\tau_{e}+\frac{S^{2} \tau_{e}^{3}}{6}\right)
$$

between $\tau, \tau_{e}$, and $S$. For arbitrary $t \geq 0$, one obtains

$$
\begin{aligned}
p_{i i}(t)= & 3 \Lambda+\frac{\Lambda S^{2} \tau_{e}^{2}}{2}+\left(S^{2} p_{22}(0)-S^{2} \Lambda\right) t^{2} e^{-\alpha t} \\
& -\left(2 S p_{12}(0)+2 \frac{S^{2}}{\alpha} \Lambda\right) t e^{-\alpha t},
\end{aligned}
$$

which fulfills the requirement that $\forall t \geq 0: p_{i i}(t)=3 \Lambda+\Lambda S^{2} \tau_{e}^{2} / 2$, if the initial conditions

$$
p_{22}(0)=\Lambda \text { and } p_{12}(0)=-\frac{S}{\alpha} \Lambda
$$

are applied.

As a concrete case, we consider argon at $1 \mathrm{~atm}$ and $300 \mathrm{~K}$, which at equilibrium has a density of $1.449 \mathrm{~kg} / \mathrm{m}^{3}$ and a dynamic viscosity of $\mu=\tau \rho \theta \mathrm{k} / \mathrm{m}=3.77 \times 10^{-5} \mathrm{~Pa}$ s. Furthermore, an argon molecule has a mass $m$ of $6.633521355393869 \times 10^{-23} \mathrm{~kg}$, and therefore the relaxation time scale becomes $\tau=4.167 \times 10^{-7} \mathrm{~s}$. Here, we choose $S=\alpha / 2=\tau_{e}^{-1}=7 /(6 \tau)$, which fulfills condition (64) and results in strong nonequilibrium solutions. The initial condition is chosen as

$$
p(0)=\frac{\Lambda}{4}\left[\begin{array}{ccc}
5 & -2 & 0 \\
-2 & 4 & 0 \\
0 & 0 & 5
\end{array}\right]
$$

and relaxes to

$$
\lim _{t \rightarrow \infty} p(t)=\frac{\Lambda}{4}\left[\begin{array}{ccc}
6 & -2 & 0 \\
-2 & 4 & 0 \\
0 & 0 & 4
\end{array}\right]
$$

\section{B. Numerical scheme}

For the numerical studies, ensembles of $N$ particles with index $k$ and initial random velocities

$$
\begin{aligned}
& V_{1}^{(k)}=\frac{\sqrt{\Lambda / \rho}}{2} \sqrt{5} \xi_{1}^{(k)}, \\
& V_{2}^{(k)}=\frac{\sqrt{\Lambda / \rho}}{2}\left(\sqrt{\frac{16}{5}} \xi_{2}^{(k)}-\sqrt{\frac{4}{5}} \xi_{1}^{(k)}\right),
\end{aligned}
$$

and

$$
V_{3}^{(k)}=\frac{\sqrt{\Lambda / \rho}}{2} \sqrt{5} \xi_{3}^{(k)}
$$

are generated (the superscript $(k)$ will be omitted in the following derivation). Note that their statistics is consistent with $\boldsymbol{p}(0)$. In order to study the bias error of $\overline{\boldsymbol{p}}^{N}(t), \overline{\boldsymbol{U}}^{N}$ is not explicitly set to zero, i.e., equation

$$
d V_{i}=-\left(\frac{\delta_{i l}}{\tau_{e}}+A_{i l}\right)\left(V_{l}-\bar{U}_{l}^{N}\right) d t+\sqrt{\frac{2 \bar{\Lambda}^{N}}{\rho \tau_{e}}} d W_{i}
$$

with

$$
\bar{\Lambda}^{N}=\frac{\bar{p}_{l l}^{N}}{3} \frac{\tau_{e}}{\tau}
$$

is solved. Furthermore, to eliminate time stepping errors, the integration schemes

$$
\begin{aligned}
V_{1}^{n+1}-V_{1}^{n}= & \left(\bar{U}_{1}^{N}-V_{1}^{n}\right)\left(1-e^{-\Delta t / \tau_{e}}\right)+\left(\bar{U}_{2}^{N}-V_{2}^{n}\right) S \Delta t e^{-\Delta t / \tau_{e}} \\
& +\sqrt{\mathscr{B}-\frac{\mathscr{C}^{2}}{\mathscr{A}}} \xi_{1}+\mathscr{C} \sqrt{\frac{1}{\mathscr{A}}} \xi_{2}, \\
V_{2}^{n+1}-V_{2}^{n}= & \left(\bar{U}_{2}^{N}-V_{2}^{n}\right)\left(1-e^{-\Delta t / \tau_{e}}\right)+\sqrt{\mathscr{A}} \xi_{2},
\end{aligned}
$$

and

with

$$
V_{3}^{n+1}-V_{3}^{n}=\left(\bar{U}_{3}^{N}-V_{3}^{n}\right)\left(1-e^{-\Delta t / \tau_{e}}\right)+\sqrt{\mathscr{A}} \xi_{3}
$$

$$
\begin{gathered}
\mathscr{A}=\frac{\bar{\Lambda}^{N}}{\rho}\left(1-e^{-2 \frac{\Delta t}{\tau_{e}}}\right), \\
\mathscr{B}=\frac{\bar{\Lambda}^{N}}{\rho} \frac{S^{2} \tau_{e}^{2}}{2}\left(1-\left(\frac{2 \Delta t^{2}}{\tau_{e}^{2}}+\frac{2 \Delta t}{\tau_{e}}+1\right) e^{-2 \frac{\Delta t}{\tau_{e}}}\right)+\mathscr{A}
\end{gathered}
$$

and

$$
\mathscr{C}=\frac{\bar{\Lambda}^{N}}{\rho} \frac{S \tau_{e}}{2}\left(\left(\frac{2 \Delta t}{\tau_{e}}+1\right) e^{-2 \frac{\Delta t}{\tau_{e}}}-1\right)
$$

are employed; its derivation is found in the Appendix. Note that the scheme (75) and (74) is statistically exact for frozen $\overline{\boldsymbol{U}}^{N}$ and $\overline{\boldsymbol{p}}^{N}$.

\section{Bias error investigations}

The $N$ particles are evolved according to Eqs. (75) and (74), and in order to achieve steady state solutions with EWTA, which are independent of the initial conditions, the parameters $\boldsymbol{U}$ and $\Lambda$ were estimated as

$$
\boldsymbol{U}=\frac{9}{10} \overline{\boldsymbol{U}}^{N}
$$

and

$$
\Lambda=\frac{9}{10}\left(\frac{\bar{p}_{l l}^{N}}{3} \frac{\tau_{e}}{\tau}\right)+\frac{1}{10}\left(\frac{\rho \theta_{r e f} k}{m} \frac{\tau_{e}}{\tau}\right) .
$$




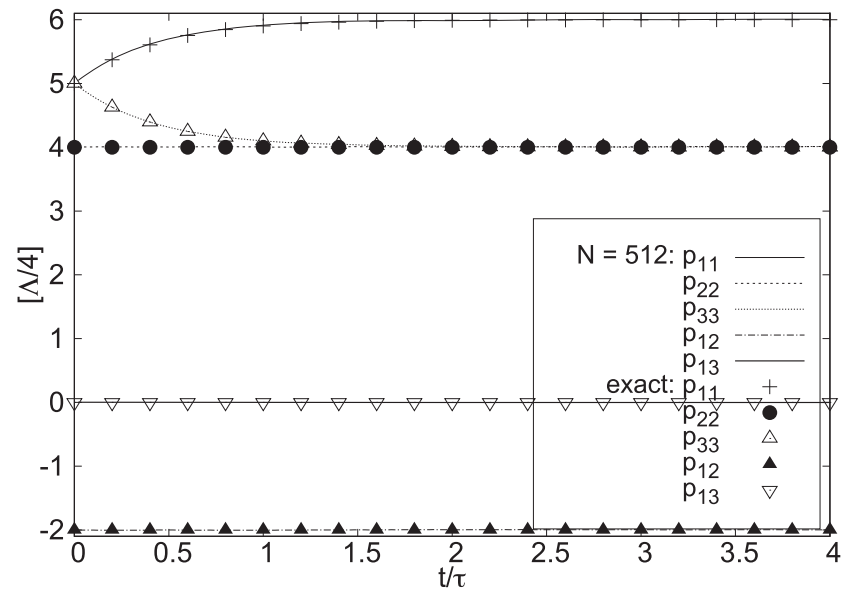

FIG. 1. Molecular stress components $p_{11}, p_{22}, p_{33}, p_{12}$, and $p_{13}$ (normalized by $\Lambda / 4$ ) as functions of $t / \tau$. The lines show the result of the particle algorithm with $N=512$ and $d t=\tau / 50$ (averaged over 8'192 independent simulations), and the symbols represent the analytical solution.

Note that these choices are consistent with the analytical solution, which predicts that $\boldsymbol{U}(t) \equiv 0$ and $p_{l l} \equiv 3 \rho \theta_{\text {ref }} \mathrm{k} / \mathrm{m}$. The numerical results, however, are subject to bias errors.

Figure 1 shows the evolution of the molecular stress components $p_{11}, p_{22}, p_{33}, p_{12}$, and $p_{13}$ (normalized by $\Lambda / 4$ ). It can be observed that the result of the particle algorithm with $N$ $=512$ and $d t=\tau / 50$ (lines; averaged over $8^{\prime} 192$ independent simulations) and the analytical solution (symbols) are almost identical.

The lines in Fig. 2 show the stress components $p_{11}$ and $p_{33}$ computed by the particle algorithm with $N \in\{2,8,32,128,512\}$ and $d t$ $=\tau / 50$. Since the data represent values averaged over a huge number

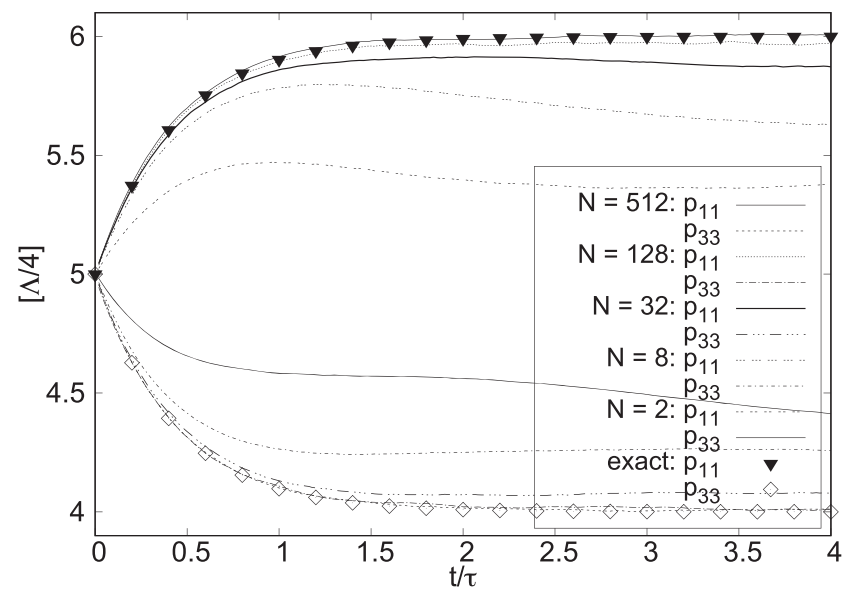

FIG. 2. Molecular stress components $p_{11}$ and $p_{33}$ (normalized by $\Lambda / 4$ ) as functions of $t / \tau$. The lines show the result of the particle algorithm with $N \in\{2,8,32,128$, $512\}$ and $d t=\tau / 50$ (averaged over $4^{\prime} 194^{\prime} 304 / N$ independent simulations), and the symbols represent the analytical solution.

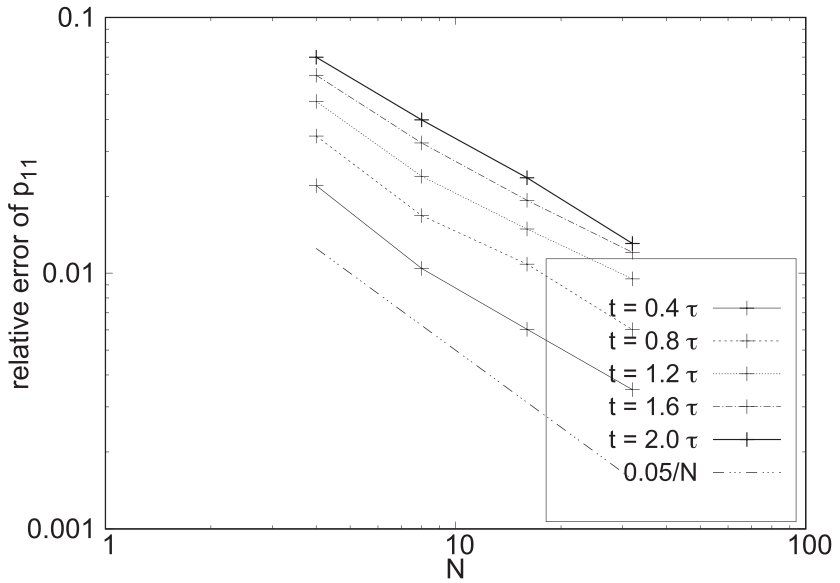

FIG. 3. Relative bias errors of $p_{11}$ at different times $t \in\{0.4 \tau, 0.8 \tau, 1.2 \tau, 1.6 \tau$, $2.0 \tau$ \} as functions of $N$.

$\left(4^{\prime} 194^{\prime} 304 / N\right)$ of independent simulations with different random seeds, their standard deviations are tiny, which is the reason that no error bars are shown. Note that the deviation from the analytical solution (symbols) represents the bias error, which becomes smaller for increasing $N$. The convergence of the relative bias error of $p_{11}$, i.e., of

$$
\frac{\bar{p}_{11}^{N}-1.5 \Lambda}{1.5 \Lambda}
$$

at different times $t \in\{0.4 \tau, 0.8 \tau, 1.2 \tau, 1.6 \tau, 2.0 \tau\}$ is shown in the plot of Fig. 3. It can be observed that it scales with approximately $N^{-1}$.

Figures 4 and 5 show the effect of EWTA for $N=2$ and $N=8$, respectively. In both cases, $d t$ was equal to $\tau$ and time averaging constants of $T=0, T=50 \tau$, and $T=200 \tau$ were used. It can be seen

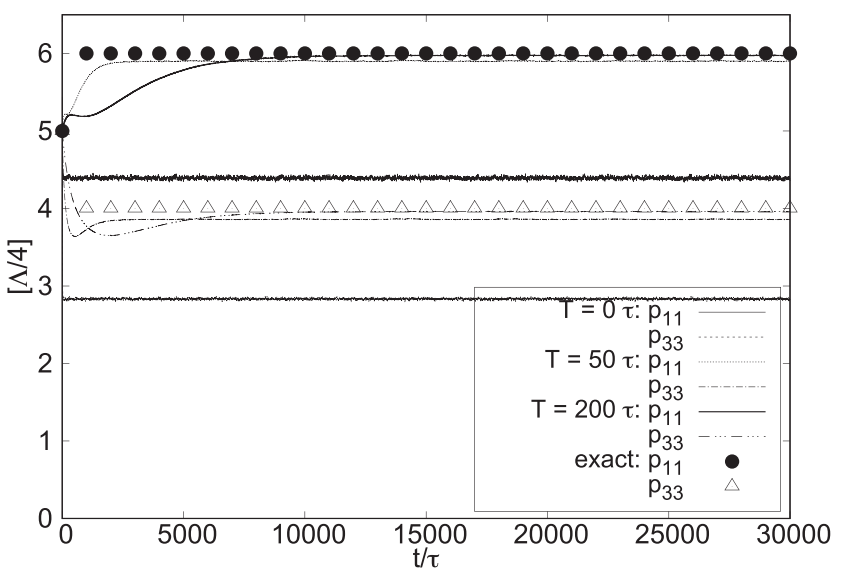

FIG. 4. Molecular stress components $p_{11}$ and $p_{33}$ (normalized by $\Lambda / 4$ ) as functions of $t / \tau$. The lines show the result of the particle algorithm with $N=2, d t=\tau$ and a time averaging constant $T \in\{0 \tau, 50 \tau, 200 \tau\}$ (averaged over 131'072 independent simulations), and the symbols represent the analytical solution. 


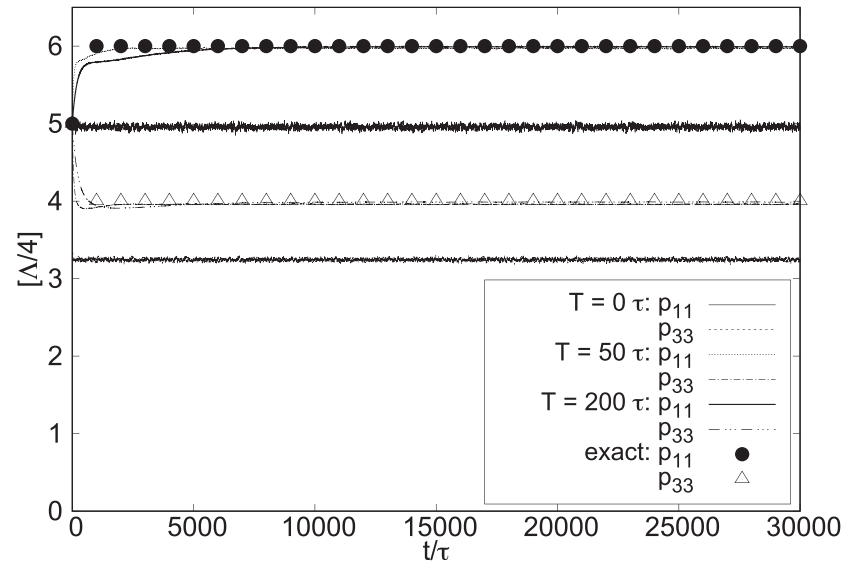

FIG. 5. Molecular stress components $p_{11}$ and $p_{33}$ (normalized by $\Lambda / 4$ ) as functions of $t / \tau$. The lines show the result of the particle algorithm with $N=8, d t=\tau$, and a time averaging constant $T \in\{0 \tau, 50 \tau, 200 \tau\}$ (averaged over $32^{\prime} 768$ independent simulations), and the symbols represent the analytical solution.

that even for such small particle numbers the numerical steady state values of $p_{11}$ and $p_{33}$ (lines; averaged over 131' 072 independent simulations) converge to the analytical solution (symbols). This can also be observed (for $N=2$ and $N=8$ ) in the plot of Fig. 6, which shows the relative bias error of $p_{11}$ at steady state, i.e.,

$$
\frac{1}{20^{\prime} 000 \tau} \int_{10^{\prime} 000 \tau}^{30^{\prime} 000 \tau}\left(\frac{\bar{p}_{11}^{N, \mu}-1.5 \Lambda}{1.5 \Lambda}\right) d t,
$$

as function of the time averaging constant $T=-\Delta t / \ln (\mu)$. Since the data points represent time averaged values over the last $20^{\prime} 000$ time steps, their standard deviation is tiny and therefore no error bars are shown. One can see that the bias error scales with approximately $T^{-1}$. It has to be mentioned that EWTA was also applied for $P_{i j}$, although the analysis of Sec. III suggests that better estimates are

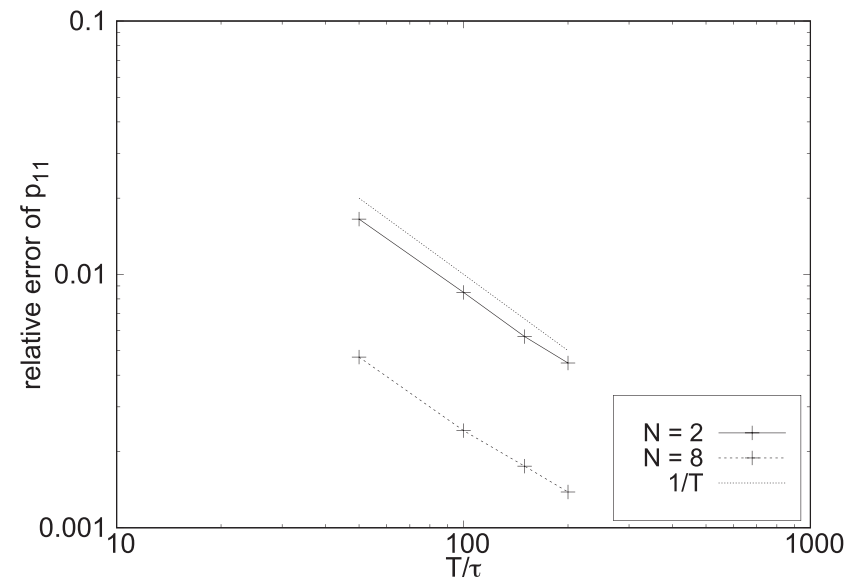

FIG. 6. Relative bias errors of $p_{11}$ at steady state for $N=2$ and $N=8$ as functions of $T / \tau$. only necessary for $U_{i}$. The reason for this is that $\bar{P}_{i j}^{N}-\rho \bar{U}_{i}^{N, \mu} \bar{U}_{j}^{N, \mu}$ can become negative, while $\bar{P}_{i j}^{N, \mu}-\rho \bar{U}_{i}^{N, \mu} \bar{U}_{j}^{N, \mu} \geq 0$ by construction.

\section{CONCLUSIONS}

The bias error of the Fokker-Planck method for rarefied gas dynamics simulations was analyzed, and as an effective mean to reduce it, exponentially weighted time averaging was explored. For these investigations, an analytical reference solution of a uniform shear flow with dissipation was derived, and in order to exclude time stepping errors, a statistically exact particle integration scheme was devised for this case. Furthermore, to reduce the statistical error to an insignificant level, the numerical results were averaged over large numbers of independent simulations. It is shown that the bias error converges with $N^{-1}$ and $T^{-1}$. It has to be noted here that for DSMC, this technique only helps to reduce the statistical error but not the bias error. In terms of memory requirement for Fokker-Planck simulations, it is very encouraging that even with extremely small particle numbers, converged results can be obtained. Moreover, the asymptotic scaling of the bias error suggest that Richardson extrapolation may be used to obtain nearly bias free solutions from two simulations with small, but different particle numbers.

\section{APPENDIX: PARTICLE INTEGRATION SCHEME}

To derive the particle integration schemes (74)-(76) presented in Sec. V B, we first convince ourselves that according to Eq. (72), the expectation of $\boldsymbol{V}^{n+1}$ conditional on $\boldsymbol{V}^{n}$ is

$$
\begin{aligned}
\mathbb{E}\left[\boldsymbol{V}^{n+1} \mid \boldsymbol{V}^{n}\right]= & \overline{\boldsymbol{U}}^{N}\left(1-e^{-\Delta t / \tau_{e}}\right)+\boldsymbol{V}^{n} e^{-\Delta t / \tau_{e}} \\
& +\underbrace{\left(\begin{array}{lll}
0 & S & 0 \\
0 & 0 & 0 \\
0 & 0 & 0
\end{array}\right)}_{\boldsymbol{A}}\left(\overline{\boldsymbol{U}}^{N}-\boldsymbol{V}^{n}\right) \Delta t e^{-\Delta t / \tau_{e}} .
\end{aligned}
$$

Note that a perturbation $\delta \boldsymbol{V}^{h}$ of $\boldsymbol{V}$ at time $t^{h} \in\left[t^{n}, t^{n+1}\right]$ has an additive effect of

$$
\delta \boldsymbol{V}^{n+1}=\delta \boldsymbol{V}^{h} e^{-\left(t^{n+1}-t^{h}\right) / \tau_{e}}-\boldsymbol{A} \delta \boldsymbol{V}^{h}\left(t^{n+1}-t^{h}\right) e^{-\left(t^{n+1}-t^{h}\right) / \tau_{e}}
$$

on $V^{n+1}$. Furthermore, note that one can replace

$$
\int_{t^{n}}^{t^{n+1}} d \boldsymbol{W}\left(t^{\prime}\right) d t^{\prime}
$$

with

$$
\lim _{n^{\prime} \rightarrow \infty} \sqrt{\frac{\Delta t}{n^{\prime}}} \sum_{h=1}^{n^{\prime}} \xi_{h}^{\prime},
$$

where $\boldsymbol{\xi}_{h}^{\prime}$ are vectors of independent, normally distributed random variables with zero mean and a variance of one. Therefore, the effect of the diffusion term in Eq. (72) on $\boldsymbol{V}^{n+1}$ during the previous time step is the same as that of perturbations of strength

$$
\sqrt{\frac{2 \bar{\Lambda}^{N}}{\rho \tau_{e}}} \sqrt{\frac{\Delta t}{n^{\prime}}} \xi_{h}^{\prime}
$$


occurring at a frequency of $n^{\prime} / \Delta t$, and one can write

$$
\begin{aligned}
\boldsymbol{V}^{n+1}= & \overline{\boldsymbol{U}}^{N}\left(1-e^{-\Delta t / \tau_{e}}\right)+\boldsymbol{V}^{n} e^{-\Delta t / \tau_{e}}+\boldsymbol{A}\left(\overline{\boldsymbol{U}}^{N}-\boldsymbol{V}^{n}\right) \Delta t e^{-\Delta t / \tau_{e}} \\
& +\sqrt{\frac{2 \bar{\Lambda}^{N}}{\rho \tau_{e}}} \lim _{n^{\prime} \rightarrow \infty} \sqrt{\frac{\Delta t}{n^{\prime}}} \sum_{h=1}^{n^{\prime}} \boldsymbol{\xi}_{h}^{\prime} e^{-\left(t^{n+1}-t^{h}\right) / \tau_{e}} \\
& -\sqrt{\frac{2 \bar{\Lambda}^{N}}{\rho \tau_{e}}} \lim _{n^{\prime} \rightarrow \infty} \sqrt{\frac{\Delta t}{n^{\prime}}} \sum_{h=1}^{n^{\prime}} \boldsymbol{A} \boldsymbol{\xi}_{h}^{\prime}\left(t^{n+1}-t^{h}\right) e^{-\left(t^{n+1}-t^{h}\right) / \tau_{e}} .
\end{aligned}
$$

From this expression, one gets

$$
\begin{aligned}
\mathbb{E}\left[V_{i}^{n+1} V_{j}^{n+1} \mid \boldsymbol{V}^{n}\right]= & \mathbb{E}\left[V_{i}^{n+1} \mid \boldsymbol{V}^{n}\right] \mathbb{E}\left[V_{j}^{n+1} \mid \boldsymbol{V}^{n}\right] \\
& +\frac{2 \bar{\Lambda}^{N}}{\rho \tau_{e}} \int_{0}^{\Delta t}\left(\delta_{i j}-S\left(\delta_{i 1} \delta_{2 j}+\delta_{i 2} \delta_{1 j}\right) t^{\prime}\right. \\
& \left.+S^{2} \delta_{i 1} \delta_{1 j} t^{\prime 2}\right) e^{-2 t^{\prime} / \tau_{e}} d t^{\prime}
\end{aligned}
$$

and after evaluating the integrals, one obtains

$$
\begin{aligned}
\mathbb{E}\left[V_{i}^{n+1} V_{j}^{n+1} \mid \boldsymbol{V}^{n}\right]= & \mathbb{E}\left[V_{i}^{n+1} \mid \boldsymbol{V}^{n}\right] \mathbb{E}\left[V_{j}^{n+1} \mid \boldsymbol{V}^{n}\right] \\
& +\frac{\bar{\Lambda}^{N}}{\rho}\left(\delta_{i j}\left(1-e^{-2 \Delta t / \tau_{e}}\right)\right. \\
& +S\left(\delta_{i 1} \delta_{2 j}+\delta_{i 2} \delta_{1 j}\right) \frac{\tau_{e}}{2}\left(\left(\frac{2 \Delta t}{\tau_{e}}+1\right) e^{-2 \Delta t / \tau_{e}}-1\right) \\
& \left.+S^{2} \delta_{i 1} \delta_{1 j} \frac{\tau_{e}^{2}}{2}\left(1-\left(\frac{2 \Delta t^{2}}{\tau_{e}^{2}}+\frac{2 \Delta t}{\tau_{e}}+1\right) e^{-2 \Delta t / \tau_{e}}\right)\right),
\end{aligned}
$$

which is consistent with the schemes (74)-(76) together with the expressions given by Eqs. (77)-(79).

\section{REFERENCES}

${ }^{1} \mathrm{P}$. Jenny, M. Torrilhon, and S. Heinz, "A solution algorithm for the fluid dynamic equations based on a stochastic model for molecular motion," J. Comput. Phys. 229, 1077-1098 (2010).

${ }^{2}$ H. Gorji, M. Torrilhon, and P. Jenny, "Fokker-Planck model for computational studies of monatomic rarefied gas flows," J. Fluid Mech. 680, 574-601 (2011).

${ }^{3}$ J. E. Broadwell, "Study of rarefied shear flow by the discrete velocity method," J. Fluid Mech. 19, 401-414 (1964).

${ }^{4}$ I. M. Gamba and S. H. Tharkabhushanam, "Spectral-Lagrangian methods for collisional models of non-equilibrium statistical states," J. Comput. Phys. 228, 2012-2036 (2009).

${ }^{5}$ L. Wu, C. White, T. J. Scanlon, J. M. Reese, and Y. Zhang, "Deterministic numerical solutions of the Boltzmann equation using the fast spectral method," J. Comput. Phys. 250, 27-52 (2013).

${ }^{6} \mathrm{~L}$. Wu, J. Zhang, J. M. Reese, and Y. Zhang, "A fast spectral method for the Boltzmann equation for monatomic gas mixtures," J. Comput. Phys. 298, 602-621 (2015).

${ }^{7}$ B. J. Alder and T. E. Wainwright, "Studies in molecular dynamics. I. General method," J. Chem. Phys. 31, 459-466 (1959).

${ }^{8} \mathrm{G}$. A. Bird, "Breakdown of translational and rotational equilibrium in gaseous expansions," AIAA J. 8, 1998-2003 (1970).

${ }^{9}$ G. A. Bird, Molecular Gas Dynamics and the Direct Simulation of Gas Flows (Oxford University Press, New York, 1994).

${ }^{10}$ G. Bird, "Sophisticated DSMC," in Notes Prepared for a Short Course at the DSMC07 Meeting, Santa Fe, USA, 2007.

${ }^{11}$ G. Bird, The DSMC Method (CreateSpace Independent Publishing Platform, 2013).
${ }^{12}$ S. Plimpton and M. Gallis, SPARTA direct simulation Monte Carlo (DSMC) simulator, Sandia National Laboratories, USA, 2015, see http://sparta.sandia.gov. ${ }^{13}$ G. Bird, M. Gallis, J. Torczynski, and D. Rader, "Accuracy and efficiency of the sophisticated direct simulation Monte Carlo algorithm for simulating noncontinuum gas flows," Phys. Fluids 21, 017103 (2009).

${ }^{14} \mathrm{~N}$. G. Hadjiconstantinou, "Analysis of discretization in the direct simulation Monte Carlo," Phys. Fluids 12, 2634-2638 (2000).

${ }^{15}$ N. G. Hadjiconstantinou, A. L. Garcia, M. Z. Bazant, and G. He, "Statistical error in particle simulations of hydrodynamic phenomena," J. Comput. Phys. 187, 274297 (2003).

${ }^{16} \mathrm{~J}$. G. Kirkwood, "The statistical mechanical theory of transport processes I. General theory," J. Chem. Phys. 14, 180 (1946).

${ }^{17}$ J. G. Kirkwood, F. P. Buff, and M. S. Green, "The statistical mechanical theory of transport processes. III. The coefficients of shear and bulk viscosity of liquids," J. Chem. Phys. 17, 988 (1949)

${ }^{18}$ J. L. Lebowitz, H. L. Frisch, and E. Helfand, "Nonequilibrium distribution functions in a fluid," Phys. Fluids 3, 325 (1960).

${ }^{19} \mathrm{H}$. Gorji, "Fokker-Planck solution algorithm for rarefied gas flows and applications of complex gas-surface interactions," ETH dissertation (ETH Zürich, 2014).

${ }^{20} \mathrm{H}$. Gorji and P. Jenny, "An efficient particle Fokker-Planck algorithm for rarefied gas flows," J. Comput. Phys. 262, 325 (2014).

${ }^{21}$ S. K. Singh, C. Thantanapally, and S. Ansumali, "Gaseous microflow modeling using the Fokker-Planck equation," Phys. Rev. E 94, 063307 (2016).

${ }^{22} \mathrm{H}$. Gorji and P. Jenny, "A device concept for demixing of gas species based on excitation of internal energy modes," in Proceedings of the ASME 2013 11th International Conference on Nanochannels, Microchannels, and Minichannels (ASME, 2013).

${ }^{23} \mathrm{H}$. Gorji and P. Jenny, "Continuous stochastic equations for diatomic rarefied gas flows," in Proceedings of the 3rd GASMEMS Workshop, Bertinoro, June 9-11, 2011.

${ }^{24} \mathrm{H}$. Gorji and P. Jenny, "A Fokker-Planck based kinetic model for diatomic rarefied gas flows," Phys. Fluids 25, 062002 (2013).

${ }^{25} \mathrm{H}$. Gorji and P. Jenny, "A kinetic model for gas mixtures based on FokkerPlanck equation,” J. Phys.: Conf. Ser. 362, 012042 (2012).

${ }^{26} \mathrm{H}$. Gorji and P. Jenny, "A generalized stochastic solution algorithm for simulations of rarefied gas flows," in Proceedings of the 2nd European Conference on Microfluidics, Toulouse, France, 2010.

${ }^{27}$ H. Gorji, N. Andric, and P. Jenny, "Variance reduction for Fokker-Planck based particle Monte Carlo schemes," J. Comput. Phys. 295, 644 (2015).

${ }^{28} \mathrm{~N}$. Andric, H. Gorji, and P. Jenny, "Influence of the gas-surface interaction model on time-dependent rarefied gas simulations," Vacuum 128, 244 (2016).

${ }^{29}$ H. Gorji, S. Küchlin, and P. Jenny, "A hybrid Fokker-Planck-DSMC solution algorithm for the whole range of knudsen numbers," in Proceedings of the ASME 2013 11th International Conference on Nanochannels, Microchannels, and Minichannels (ASME, 2013).

${ }^{30} \mathrm{H}$. Gorji and P. Jenny, "Fokker-Planck-DSMC algorithm for simulations of rarefied gas flows," J. Comput. Phys. 287, 110 (2015).

${ }^{31}$ S. Küchlin and P. Jenny, "Parallel Fokker-Planck-DSMC algorithm for rarefied gas flow simulation in complex domains at all Knudsen numbers," J. Comput. Phys. 328, 258-277 (2017).

${ }^{32}$ S. Küchlin and P. Jenny, "Automatic mesh refinement and parallel load balancing for Fokker-Planck-DSMC algorithm," J. Comput. Phys. 363, 140-157 (2018).

${ }^{33}$ S. Küchlin, "Analysis and parallel implementation of the Fokker-Planck-DSMC algorithm," Ph.D. thesis, ETH Zurich, 2018.

${ }^{34}$ P. Jenny, S. B. Pope, M. Muradoglu, and D. A. Caughey, "A hybrid algorithm for the joint PDF equation of turbulent reactive flows," J. Comput. Phys. 166, 218-252 (2001).

${ }^{35}$ M. Muradoglu, P. Jenny, S. B. Pope, and D. A. Caughey, "A consistent hybrid finite-volume/particle method for the PDF equations of turbulent reactive flows," J. Comput. Phys. 154, 342-371 (1999).

${ }^{36} \mathrm{M}$. Pfeiffer and M. H. Gorji, "Adaptive particle-cell algorithm for FokkerPlanck based rarefied gas flow simulations," Comput. Phys. Commun. 213, 1-8 (2017). 
${ }^{37}$ P. Jenny, M. Muradoglu, K. Liu, S. B. Pope, and D. A. Caughey, "PDF simulations of a bluff-body stabilized flow," J. Comput. Phys. 169, 1-23 (2001).

${ }^{38}$ S. Rjasanow and W. Wagner, Stochastic Numerics for the Boltzmann Equation (Springer, 2005).

${ }^{39}$ M. H. Gorji, "Fokker-Planck solution algorithm for rarefied gas flows and applications of complex gas-surface interactions," Ph.D. thesis, ETH Zurich, 2014.

${ }^{40}$ H. Risken, "Fokker-Planck equation," in The Fokker-Planck Equation (Springer, 1996), pp. 63-95.

${ }^{41}$ C. W. Gardiner et al., Handbook of Stochastic Methods (Springer Berlin, 1985), Vol. 3.
${ }^{42}$ E. Jun, M. H. Gorji, M. Grabe, and K. Hannemann, "Assessment of the cubic Fokker-Planck-DSMC hybrid method for hypersonic rarefied flows past a cylinder," Comput. Fluids 168, 1-13 (2018).

${ }^{43}$ E. Josyula, R. Arslanbekov, V. Kolobov, and S. F. Gimelshein, "Evaluation of kinetic/continuum solver for hypersonic nozzle-plume flow," J. Spacecr. Rockets 45, 665-676 (2008).

${ }^{44} \mathrm{C}$. Cercignani and S. Cortese, "Validation of a Monte Carlo simulation of the plane Couette flow of a rarefied gas,"J. Stat. Phys. 75, 817 (1994).

${ }^{45} \mathrm{~V}$. Garzo and A. Santos, Kinetic Theory of Gases in Shear Flows: Nonlinear Transport (Springer, 2003). 